\title{
A Case-Based Reasoning Approach to Provide Adaptive Feedback in Microworlds
}

\author{
Sergio Gutierrez-Santos, Mihaela Cocea, and George Magoulas* \\ Birkbeck College, London Knowledge Lab, University of London \\ 23-29 Emerald St, WC1N 3QS, London (UK) \\ \{sergut,mihaela,gmagoulas\}@dcs.bbk.ac.uk
}

\begin{abstract}
This paper presents a case-based reasoning (CBR) approach to provide adaptive support in microworlds. Interaction in microworlds is complex and unstructured, making the analysis of student behaviour difficult and the provision of computer-based feedback challenging. Our approach starts with the ellicitation of expected solutions to microworld tasks (both valid and common mistakes) to generate a case base. This is used to evaluate the actions of students and provide adapted feedback. Keywords: case-based reasoning, exploratory learning environments, microworlds, adaptive feedback
\end{abstract}

\section{Introduction}

Microworlds are a special kind of exploratory learning environments (ELE) where students are allowed to create their own models/constructions, and explore their properties and relationships. Providing appropriate support in such a situation is crucial for adequate learning [1], but this is an challenging endeavour due to the ill-definedness of the interaction. This paper proposes an approach to provide support for a microworld called eXpresser. The goal is to provide adaptive feedback on-demand to alleviate the workload of teachers in classrooms.

Most cases of analysis and support for ELE are related to systems for learning Physics [2-4]. There are also relevant works in the domain of Mathematics [5, $6]$. Although these systems grant some freedom to students, none of them allow to create new models/constructions. Our approach uses case-based reasoning to provide feedback to students interacting with a mathematical microworld that allows them to create new shapes and algebraic expressions from scratch.

\section{The eXpresser microworld}

The eXpresser is a microworld that allows students to create figural patterns and link them with expressions. Creating and combining patterns and expressions, students can create many different structures in the computer [7]. The construction of these structures in the context of a classroom task scaffolds the development of algebraic skills of the students (our target age is 11-12 years).

The microworld grants a lot of freedom to students, who may construct their patterns in a multitude of ways, that range from valid ones (see Figure 1) to

\footnotetext{
* The authors would like to acknowledge the rest of the members of the MiGen team

This work is funded by TLRP (e-Learning Phase-II, RES-139-25-0381).
} 
off-task behaviour. The complexity and variety of possible approaches makes it impossible to list all of them. Our approach is based on the identification of the main possibilities with the help of pedagogy experts, using this initial case base to judge the specific approaches of students and provide adapted feedback.

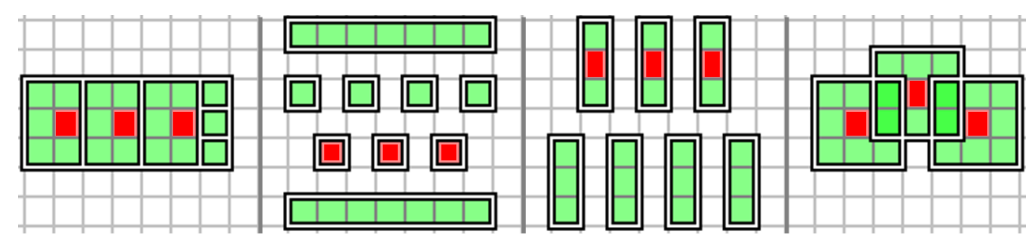

(a)

(b)

(c)

(d)

Fig. 1. Several examples of expected solutions for a 'footpath' task. Each solution can be constructed in many different ways (i.e. different actions lead to similar structures). The internal structure of the patterns is highlighted here for clarity. In eXpresser all constructions would look the same in the normal course of the task.

\section{CBR for adapted feedback generation}

The most important containers of information in a CBR system are the cases themselves, each of them storing information about one problem and its solution. The other knowledge containers are the similarity measures (used to compare cases) and the adaptation mechanism (used to adapt solutions to a new problem). In our approach, problems are possible construction strategies on the microworld and solutions are feedback provided to learners on demand (see Figure 2). Construction strategies are represented as series of shapes. Shapes are defined by attributes such as position, colour, and relations to other shapes (e.g. there are as many green tiles as five times the number of red tiles).

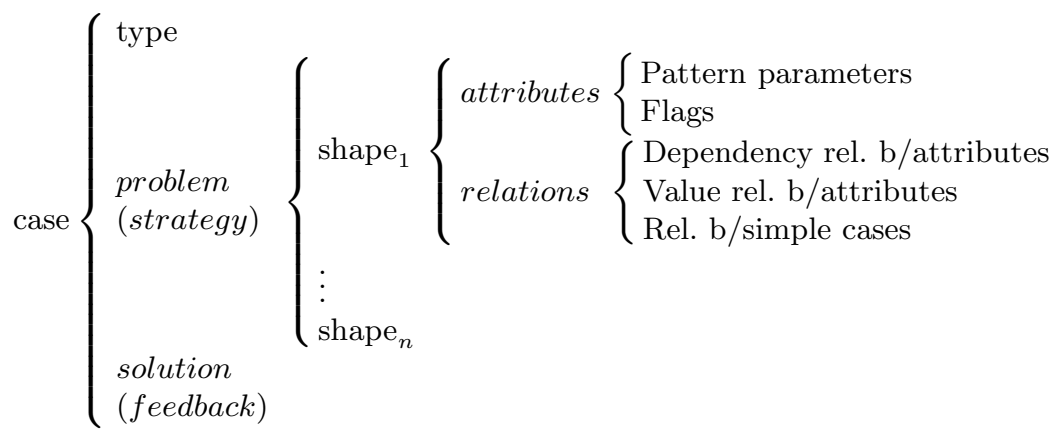

Fig. 2. Case structure. For a more detailed description of strategies and shapes, see [8].

When a learner working with the microworld asks for help, the learner's construction is processed into a new problem, i.e. strategy. This strategy is compared with all the strategies in the case base to find the most similar one. Details about the similarity metrics used can be found in [8]. 
If a perfect match is found, feedback is immediately generated as the solution of the matching case. Otherwise, the case solution will need to be adapted to generate the feedback. There are two types of cases in our approach, so there are two different adaptation processes.

If the retrieved case is an "expected solution" (i.e. the student is probably working in the right direction), the text of the feedback changes to give the student an encouraging message and additional information is put in place to highlight those aspects on which the student should reflect upon. This information can be extracted from the similarity comparison between the case and the students result. If the retrieved case can be a "common mistake" (i.e. the student has a misconception frequently observed in practice according to pedagogy experts) the feedback provided contains a common message developed by the pedagogical team. This message makes the students reflect on past actions and realise their misconception. In this case, there is usually no further adaptation of the feedback because it is already specifically targeted towards one well-known misconception. Lastly, it can happen that the student's construction is not similar to any of the problems in the case base (e.g. the student has made little progress on the task, or she might have found a new perfectly valid approach, which had not been considered by the design team). This situation is beyond the scope of the system, so a message is handled to the human teacher.

The proposed approach makes it possible to provide adapted feedback in microworlds, where the unstructured nature of the interaction poses an important challenge. We plan to extend the approach to other microworlds.

\section{References}

1. Kirschner, P., Sweller, J., Clark, R.: Why minimal guidance during instruction does not work:an analysis of the failure of constructivist, discovery, problem-based, experiential, \& inquiry-based teaching. Educational Psychologist 41 (2006) 75-86

2. vanLehn, K., Lynch, C., Schultz, K., Shapiro, J., Shelby, R., Taylor, L., Treacy, D., Weinstein, A., Wintersgill, M.: The ANDES physics tutoring system: Lessons learned. Int. Journal of Artificial Intelligence and Education 15 (2005)

3. Veermans, K., van Joolingen, W.R.: Combining heuristics and formal methods in a tool for supporting simulation-based discovery learning. In: Intelligent Tutoring Systems, Springer 217-226

4. Stathacopoulou, R., Magoulas, G., Grigoriadou, M., Samarakou, M.: Neuro-fuzzy knowledge processing in intelligent learning environments for improved student diagnosis. Information Sciences 170 (2005) 273-307

5. Bunt, A., Conati, C.: Probabilistic student modelling to improve exploratory behaviour. User Modeling and User-Adapted Interaction 13 (August 2003) 269-309

6. Mavrikis, M., Lee, J.: Towards contingent and affective microworlds. In: Int. Conf. in Artificial Intelligent in Education. (2003)

7. Pearce, D., Geraniou, E., Mavrikis, M., Gutierrez-Santos, S., Kahn, K.: Using pattern construction and analysis in an exploratory learning environment for understanding mathematical generalisation: The potential for intelligent support. In: Int. Workshop on Intelligent Support for Exploratory Environments. (2008)

8. Cocea, M., Gutierrez-Santos, S., Magoulas, G.: Enhancing modelling of users strategies in exploratory learning through case-base maintenance. In: Workshop on Casebased Reasoning, SGAI Int. Conf. on Artificial Intelligence. (2009) 